\title{
APPLICATION OF THE METHOD OF INTEGRAL EQUATION FOR CALCULATING A STEP-TRANSITION IN A COAXIAL WAVEGUIDE
}

\author{
V.M. Morozov, V.I. Magro* \\ Oles Honchar Dnipro National University, Dnipro, Ukraine \\ *e-mail:magrov@i.ua
}

The stepwise transition in the coaxial waveguide is calculated by the integral equation method. To solve the problem, the entire region of field definition is conditionally divided into three partial areas for which the field components are recorded. A system of equations is obtained that allows one to calculate the reflection coefficient of a $\mathrm{T}$-wave from this homogeneity. The geometric dimensions of the waveguide, which provide the minimum value of the reflection coefficient, are given.

Keywords: coaxial waveguide, step heterogeneity, integral equation, method, reflection coefficient.

Received 05.07.2020; Received in revised form 20.08.2020; Accepted 04.09.2020

\section{Introduction}

Let us consider abrupt changes in the cross-section of a coaxial line. Let the diameter of the outer conductor be not changed. In this case, the diameter of the inner conductor abruptly increases from $r_{0}$ to $r_{1}$. Then the wave impedance of the coaxial line decreases from $Z_{1}$ to $Z_{2}$. Such a section of the coaxial line can be considered as a four-terminal network, which contains two segments of the coaxial line $a_{1}$ and $a_{2}$ having wave impedances $Z_{1}$ and $Z_{2}$. A change in the structure of the electric field is equivalent to the inclusion of a concentrated capacitance $C$ between these segments.

Let the diameter of the inner conductor be not changed. In this case, the diameter of the outer conductor increases stepwise from $r_{2}$ to $r_{3}$. Then the wave impedance in this section decreases stepwise from $Z_{1}$ to $Z_{2}$. A change in the structure of the electric field is equivalent to the inclusion of a concentrated capacitance $C$ between these segments.

Consider a non-reflective change in the cross-section of the coaxial line. Fig. 1 shows the simplest way to maintain the constancy of the wave impedance of a line when its crosssection changes. To maintain a constant wave drag along the line, it is necessary that $\frac{r_{0}}{r_{2}}=\frac{r_{1}}{r_{3}}$. The structure of the magnetic field in the region of the diameter jump remains unchanged. The distortion of the electric field structure in the region of the size jump can be taken into account by introducing parallel capacitance $C$ into the equivalent circuit. To expand the working frequency band, the influence of stray capacitance $C$ is compensated by sequentially switching the inductive reactance. Serial inductance can be obtained by shifting the planes in which the diameters of the outer and inner conductor of the coaxial line abruptly change by an amount $l$ (Fig. 2). With this displacement, a part of the thin inner conductor falls inside the coaxial line, which has a larger diameter of the outer conductor. The value of the capacitance $C$ decreases and such an inhomogeneity of the line behave as an additional series inductance.

A number of papers consider the propagation of electromagnetic waves in an inhomogeneous coaxial waveguide [1-6]. In [1], the analysis of inhomogeneities in a coaxial waveguide was carried out on the basis of telegraph equations.

In [2], a model of the propagation of electromagnetic waves in inhomogeneous lossy coaxial cables is considered. In this paper, an asymptotic analysis of the "homogenized" telegraph model in the time domain is used.

In [3], a theoretical study of wave propagation in a nanometric coaxial waveguide in a real metal is presented. 
The electrodynamic characteristics of simple inhomogeneities in multimode circular and coaxial waveguides are considered in the paper [4]. In [5-6], for the analysis of coaxial waveguides, the finite difference time domain method is used.

This review shows that there is a need to consider this heterogeneity in a coaxial waveguide using classical electrodynamics methods [7-8].

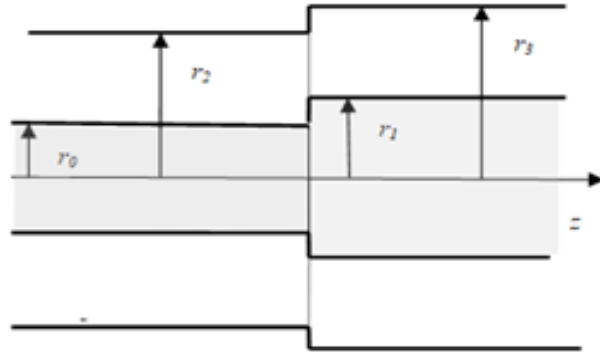

Fig.1. An abrupt change in the cross-section of the line while maintaining the value of the line impedance.

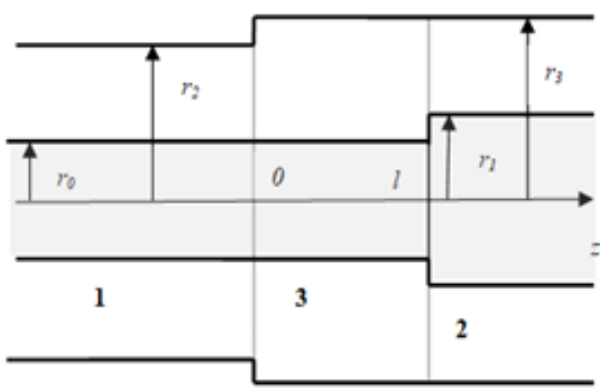

Fig. 2. An abrupt change in the cross-section of the line that does not create reflections.

\section{Formulation of the problem}

To solve the problem of electromagnetic field diffraction in a stepwise transition in a coaxial waveguide, the entire field of field determination $-\infty \leq z<0, r_{0}<\rho<r_{2}$ i $0 \leq z \leq l, r_{0}<\rho<r_{3} ; \quad l<z \leq+\infty, r_{1}<\rho<r_{3}$ is divided into partial regions $1,2,3$ (Fig. 2).

Region 1: $-\infty \leq z \leq l, r_{0}<\rho<r_{2}$. Region 2: $0<z \leq+\infty, r_{1}<\rho<r_{3}$. Region 3: $0 \leq z<l, r_{0}<\rho<r_{3}$.

In region 1 , the main $\mathrm{T}$-wave is excited at a point $z=-\infty$. From region 1 , a $\mathrm{T}$-wave falls on the inhomogeneity in the line. The walls of the stepped coaxial transition are assumed to be perfectly conducting; the medium in the line is homogeneous and isotropic. The electromagnetic field in such a system will be completely described by the $H_{\varphi}(\rho, z)$ field component.

From the Maxwell equations we can obtain the following differential equation

$$
\frac{\partial}{\partial \rho^{2}} \rho H_{\phi v}+\frac{1}{\rho} \frac{\partial}{\partial \rho} \rho H_{\phi v}+\frac{\partial^{2}}{\partial z^{2}} \rho H_{\phi V}+\kappa_{v}^{2} \rho H_{\phi v}=0
$$

where $v=1,2,3$ is the index that indicates the area number; $\kappa_{v}=\omega \sqrt{\varepsilon \mu}$ is the wave number.

\section{Integral equations for the electromagnetic field}

We solve equation (1) by the method of separation of variables $H_{\phi}(\rho, z)=Z(\rho) \phi(z)$. For a function $\varphi$ that depends on the $z$ coordinate, we obtain the following dependence: in the first region $\exp [\gamma(z-l)]$, in the second region $\exp (-\gamma z)$, where $\gamma$ is the propagation constant. When finding a function $Z(\rho)$, two cases are possible.

1. When $\gamma^{2}+\kappa^{2}=0$, we have that $\frac{1}{\rho} \frac{\partial}{\partial \rho} \rho \frac{\partial}{\partial \rho}\left(\rho H_{\phi}\right)=0 ; \rho H_{\phi}=$ const. Then we obtain for the $1^{\text {st }}$ region 


$$
Z_{1}\left(X_{10} \rho\right)=\frac{1}{\rho\left(\ln _{T_{0}}\right)^{1 / 2}}
$$

Similarly, for the $2^{\text {nd }}$ region, we can obtain

$$
Z_{1}\left(\chi_{20} \rho\right)=\frac{1}{\rho\left(\ln \frac{r_{g}}{r_{1}}\right)^{1 / 2 \pi}} .
$$

2. When $\gamma^{2}+\kappa^{2} \neq 0$, we get a solution for the $1^{\text {st }}$ region

$$
Z_{1}\left(\chi_{1 n} \rho\right)=D_{2 n}\left[N_{0}\left(\chi_{1 n} r_{0}\right) J_{1}\left(\chi_{1 n} \rho\right)-J_{0}\left(\chi_{1 n} r_{0}\right) N_{1}\left(\chi_{1 n} \rho\right)\right]
$$

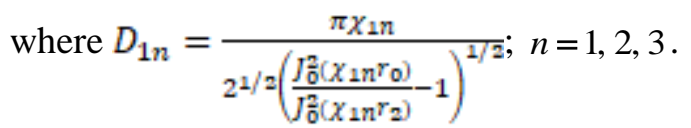

For the $2^{\text {nd }}$ region

$$
Z_{1}\left(\chi_{2 n} \rho\right)=D_{2 n}\left[N_{0}\left(\chi_{2 n} \gamma_{1}\right) J_{1}\left(\chi_{2 n} \rho\right)-J_{0}\left(\chi_{2 n} \gamma_{1}\right) N_{1}\left(\chi_{2 n} \rho\right)\right]
$$

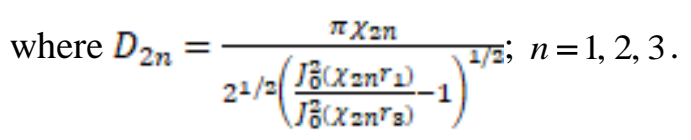

To determine the own numbers $\chi_{1 n}$, we use the equation

$$
I_{0}\left(\chi_{1 n} r_{0}\right) N_{0}\left(\chi_{1 n} r_{2}\right)-J_{0}\left(\chi_{1 n} r_{2}\right) N_{0}\left(\chi_{1 n} r_{0}\right)=0 .
$$

To apply the integral equation method, it is necessary to construct the Green functions of the selected regions. We will use the "source-like" representation for the Green function:

$$
\begin{aligned}
& G_{1}\left(\rho, z, \rho^{r}, z^{\prime}\right)=\sum_{m=0}^{\infty} Z_{m}^{(1)}(\rho) Z_{m}^{(1)}(\rho) f_{m}^{(1)}\left(z, z^{\prime}\right) ; \\
& G_{2}\left(\rho, z_{,} \rho^{*}, z^{*}\right)=\sum_{m=0}^{\infty} Z_{m}^{(2)}(\rho) Z_{m}^{(2)}\left(\rho^{\gamma}\right) f_{m}^{(2)}\left(z, z^{*}\right) ; \\
& G_{3}\left(\rho, z_{,} \rho^{*}, z^{*}\right)=\sum_{m=0}^{\infty} Z_{m}^{(3)}(\rho) Z_{m}^{(3)}\left(\rho^{\gamma}\right) f_{m}^{(3)}\left(z, z^{*}\right) .
\end{aligned}
$$

As functions $Z_{m}(\rho)$ and $Z_{m}\left(\rho^{\prime}\right)$, we choose orthonormal systems of eigenfunctions of a coaxial waveguide

$$
\begin{aligned}
& Z_{m}^{(1)}(\rho)=Z_{1}\left(\chi_{1 m} \rho\right) \\
& Z_{m}^{(2)}(\rho)=Z_{1}\left(\chi_{2 m} \rho\right) ; \\
& Z_{m}^{(3)}(\rho)=Z_{1}\left(\chi_{3 m} \rho\right) .
\end{aligned}
$$

Here $Z_{1}\left(\chi_{1 m} \rho\right)=D_{1 m}\left[N_{0}\left(\chi_{1 m} r_{0}\right) J_{1}\left(\chi_{1 m} \rho\right)-J_{0}\left(\chi_{1 m} r_{0}\right) N_{1}\left(\chi_{1 m} \rho\right)\right]$

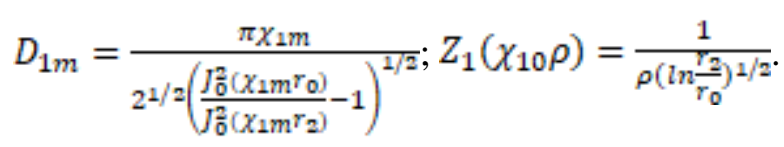

Expressions for functions $Z_{1}\left(\chi_{2 m} \rho\right)$ and $Z_{1}\left(\chi_{3 m} \rho\right)$ are got like $Z_{1}\left(\chi_{1 m} \rho\right)$. 
Functions $f_{m}\left(z, z^{\prime}\right)$ are one-dimensional Green functions of the selected regions and have the following form:

$$
\begin{aligned}
& f_{m}^{(1)}\left(z, z^{\prime}\right)=\frac{1}{\gamma_{m}}\left\{\begin{array}{ll}
e^{\gamma_{m}(z-l)} c h \gamma_{m} z^{*}, & z>z^{\prime} \\
e^{\gamma_{m}\left(z^{-}-l\right)} c h \gamma_{m} z^{\prime} & z \leq z^{\prime}
\end{array} ;\right. \\
& f_{m}^{(2)}\left(z, z^{\prime}\right)=\frac{1}{\gamma_{m}}\left\{\begin{array}{ll}
e^{\gamma_{m} z^{2}} c h \gamma_{m} z^{*}, & z>z^{\prime} \\
e^{\gamma_{m} z^{*}} c h \gamma_{m} z_{v} & z \leq z^{\prime}
\end{array}\right. \text {. } \\
& f_{m}^{(3)}\left(z, z^{\prime}\right)=\frac{1}{\gamma_{m^{s h} \gamma_{m} l}} \begin{cases}\operatorname{ch}\left[\gamma_{m}\left(z^{*}-l\right)\right] \operatorname{ch} \gamma_{m} z_{v}, & z>z^{\prime} \\
\operatorname{ch}\left[\gamma_{m}(z-l)\right] \operatorname{ch} \gamma_{m} z^{*} & z \leq z^{\prime \prime}\end{cases} \\
& \gamma_{m}=\sqrt{\chi_{m}^{2}-\kappa^{2}} ; \kappa=\frac{2 \pi}{\lambda} .
\end{aligned}
$$

Differential equation (1) can be rewritten as follows:

$$
\left(\frac{\partial}{\partial \rho^{2}}+\frac{1}{\rho} \frac{\partial}{\partial \rho}+\frac{\partial^{2}}{\partial z^{2}}+\frac{1}{\rho} K^{2}\right) H_{\phi}(\rho, z)=0 .
$$

The Green's function of a differential equation is the solution of this equation with the $\delta$ function in the right-hand side

$$
\left(\frac{\partial}{\partial \rho^{2}}+\frac{1}{\rho} \frac{\partial}{\partial \rho}+\frac{\partial^{2}}{\partial z^{2}}+\frac{1}{\rho} K^{2}\right) G\left(\rho, z, \rho^{r}, z^{\prime}\right)=\frac{\delta\left(\rho-\rho^{r}\right) \delta\left(\phi-\phi^{\prime}\right) \delta\left(z-z^{\prime}\right)}{\rho}
$$

To solve by the integral equation method, we multiply equation (2) by $G\left(\rho, z, \rho^{\prime}, z^{\prime}\right)$, and equation (3) by $H_{\varphi}(\rho, z)$. Then subtract the second equation from the third equation. We multiply the resulting equation by $\rho d \rho d \varphi d z$ and integrate over the volume $V$, then we use the second Green theorem. As a result, we obtain three integral equations for the magnetic field strength at the internal points of the selected regions:

$$
\begin{aligned}
& H_{1}(\rho, z)=H_{a x c}(\rho, z)+\int_{0}^{l}\left[G_{1}\left(\rho, z, \rho^{r}, z^{r}\right) \frac{\partial H_{3}\left(\rho^{r} z^{v}\right)}{\partial \rho^{r}}\right]_{\rho^{v}=r_{3}} \rho^{r} d z^{s}+
\end{aligned}
$$

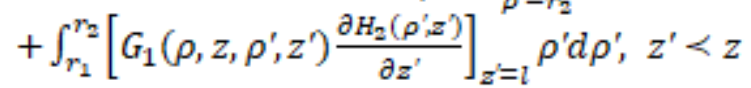

$$
\begin{aligned}
& H_{2}(\rho, z)=-\int_{0}^{l}\left[G_{2}\left(\rho, z, \rho^{r}, z^{\prime}\right) \frac{\partial H_{3}\left(\rho^{r}, z^{\prime}\right)}{\partial \rho^{r}}\right]_{\rho^{r}=r_{1}} \rho^{r} d z^{*} \\
& -\int_{r_{1}}^{r_{z}}\left[G_{2}\left(\rho, z, \rho^{r}, z^{*}\right) \frac{\partial H_{1}\left(\rho^{*}, z^{*}\right)}{\partial z^{*}}\right]_{z^{*}=0} \rho^{t} d \rho^{*} ;
\end{aligned}
$$

The system of Fredholm integral equations of the second kind (4) - (6) allows us to determine the fields in separate regions from a given external field $H_{e x c}(\rho, z)$ and Green's functions. Having eliminated the field $H_{3}(\rho, z)$ in equations (4) and (5) using (6), we obtain a system of integral equations that connect the fields in 1 and 2 regions. We will look for a solution in the form of a series expansion in eigenfunctions

$$
\begin{gathered}
H_{1}(\rho, z)=H_{\text {exc }}(\rho, z)+\sum_{k=0}^{\infty} A_{k} Z_{1}\left(\chi_{1 k} \rho\right) e^{\gamma_{1 k}(z-l)} ; \\
H_{2}(\rho, z)=+\sum_{k=0}^{\infty} B_{k} Z_{1}\left(\chi_{2 k} \rho\right) e^{\gamma_{\mathrm{ak}} z}
\end{gathered}
$$




$$
H_{e x c}(\rho, z)=2 c h \gamma_{10}(z-l) Z_{1}\left(\chi_{10} \rho\right)=\frac{2 c h \gamma_{10}(z-l)}{\rho\left(\ln \frac{T_{z}}{T_{\mathrm{n}}}\right)^{1 / 2}} .
$$

Here $A_{k}$ and $B_{k}$ are the unknown coefficients of the scattering matrix.

Let us consider equation (6) and find the derivatives $\left.\frac{\partial H_{1}\left(\rho_{z}^{r} z^{v}\right)}{\partial z^{v}}\right|_{z^{\prime}=0}$ и $\left.\frac{\partial H_{2}\left(\rho_{z}^{r} z^{v}\right)}{\partial z^{v}}\right|_{z^{z}=1}$. We substitute these derivatives and expressions for the Green's functions into equation (6). After some transformations, we obtain the expression for the magnetic field strength $H_{3}(\rho, z)$.

Let us consider equation (4) and take derivatives $\left.\frac{\partial H_{3}\left(\rho^{r} z\right)}{\partial \rho^{r}}\right|_{\rho^{v}=r_{z}}$ и $\left.\frac{\partial H_{2}\left(\rho^{r} z^{v}\right)}{\partial z^{v}}\right|_{z^{\prime}=i}$. We substitute the found derivatives and the expression for the Green's function into equation (4). After some transformations, we obtain the expression for the magnetic field strength $H_{1}(\rho, z)$. We substitute in the found expression for the magnetic field $H_{1}(\rho, z)$ equation (7), multiply the right and left sides of the resulting expression by $\rho Z_{1}\left(\chi_{1 m} \rho\right)$ and integrate from $r_{0}$ to $r_{2}$. We equate the coefficients for the same functions, thus getting the expression for $A_{p}$. Carrying out similar actions with equation (5), we obtain the expression for $B_{p}$.

Thus, the system of linear algebraic equations for the unknown coefficients of the multiwave scattering matrix has the form:

$$
\begin{aligned}
& A_{p}=-\sum_{n=0}^{\infty} \sum_{i=0}^{\infty} A_{i} \frac{\gamma_{1 i} e^{-\gamma_{1 i} l} c h l \gamma_{3 n} l}{\gamma_{3 n} \gamma_{1 p} \operatorname{sh} \gamma_{3 n} l} p_{i n} \alpha\left(\chi_{3 n} r_{2}\right) Z_{1}\left(\chi_{1 p} r_{2}\right) r_{2} \omega_{p n}- \\
& -\sum_{n=0}^{\infty} \sum_{i=0}^{\infty} B_{i}\left(\frac{\gamma_{2 i} e^{-\gamma_{3 i} l} c h \gamma_{3 n} l}{\gamma_{3 n} \gamma_{1 p} \operatorname{sh} \gamma_{3 n} l} q_{i n} \alpha\left(\chi_{3 n} r_{2}\right) Z_{1}\left(\chi_{1 p} r_{2}\right) r_{2} v_{p n}-\frac{\gamma_{2 i}}{\gamma_{1 p}} e^{-\gamma_{2 i} l} q_{p i}\right)+(9) \\
& +\sum_{n=0}^{\infty} \frac{\gamma_{10}}{\gamma_{1 p} \gamma_{3 n}} \frac{s h \gamma_{10} l c h \gamma_{3 n} l}{s h \gamma_{3 n} l} p_{0 n} \alpha\left(\chi_{3 n} r_{2}\right) r_{2} \omega_{p n} ; \\
& B_{j}=\sum_{n=0}^{\infty} \sum_{i=0}^{\infty} A_{i}\left(\frac{\gamma_{1 i} e^{-\gamma_{1 i} l} c h \gamma_{3 n} l}{\gamma_{3 n} \gamma_{2 j} \operatorname{sh} \gamma_{3 n} l} p_{i n} \alpha\left(\chi_{3 n} r_{1}\right) Z_{1}\left(\chi_{2 j} r_{1}\right) r_{1} Q_{j n}-\frac{\gamma_{1 i}}{\gamma_{2 j}} f_{i j}\right)+ \\
& +\sum_{n=0}^{\infty} \sum_{i=0}^{\infty} B_{i} \frac{\gamma_{2 i} e^{-\gamma_{2 i} l} c h \gamma_{3 n} l}{\gamma_{3 n} \gamma_{2 j} \operatorname{sh} \gamma_{3 n} l} q_{i n} \alpha\left(\chi_{3 n} r_{1}\right) Z_{1}\left(\chi_{2 j} r_{1}\right) r_{1} Q_{j n}- \\
& -\sum_{n=0}^{\infty} 2 \frac{\gamma_{10}}{\gamma_{2 j} \gamma_{3 n}} \frac{\operatorname{sh} \gamma_{10} l c h \gamma_{3 n} l}{s h \gamma_{3 n} l} p_{0 n} \alpha\left(\chi_{3 n} r_{1}\right) Z_{1}\left(\chi_{2 j} r_{1}\right) r_{1} Q_{j n}- \\
& -2 \frac{\gamma_{10}}{\gamma_{2 i}} \operatorname{ch} \gamma_{10} l f_{0 j}
\end{aligned}
$$


where

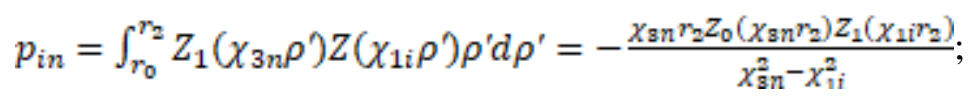

$$
\begin{aligned}
& p_{0 n}=\int_{r_{0}}^{r_{2}} Z_{1}\left(X_{3 n} \rho^{\prime}\right) Z\left(X_{10 i} \rho^{\prime}\right) \rho^{\prime} d \rho^{\prime}=-\frac{X_{3 n} r_{2} Z_{0}\left(X_{s n} r_{2}\right) Z_{1}\left(X_{10} r_{2}\right)}{x_{3 n}^{\mathrm{n}}-X_{10}^{\mathrm{n}}} \\
& \alpha\left(\chi_{3 n} r_{2}\right)=Z_{1}^{\prime}\left(\chi_{3 n} r_{2}\right)=\frac{D_{1 n}}{\chi_{3 n}}\left[N_{0}\left(\chi_{3 n} r_{0}\right)\left\{J_{1}\left(\chi_{3 n} r_{2}\right)-\frac{J_{0}\left(\chi_{3 n} r_{2}\right)}{r_{2} \chi_{3 n}}\right\}-J_{0}\left(\chi_{3 n} r_{0}\right)\left\{N_{0}\left(\chi_{3 n} r_{2}\right)-\frac{N_{1}\left(\chi_{1 m} \rho\right)}{r_{2} \chi_{3 n}}\right\}\right]
\end{aligned}
$$

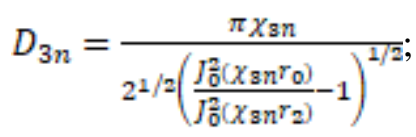

$$
\begin{aligned}
& \omega_{p n}=\int_{0}^{l} \operatorname{ch}\left[\gamma_{1 p}\left(z^{\prime}-l\right) \operatorname{ch} \gamma_{3 n} z^{\prime} d z^{*}=\frac{\gamma_{s n}}{\gamma_{\mathbb{B n}}^{\mathrm{s}}-\gamma_{1 \mathrm{p}}^{\mathrm{a}}} \operatorname{sh} \gamma_{3 n} l\right. \\
& v_{p n}=\int_{0}^{l} \operatorname{ch}\left[\gamma_{1 p}\left(z^{*}-l\right)\right] \operatorname{ch}\left[\gamma_{3 n}\left(z^{*}-l\right)\right] d z^{\prime}=\frac{s h\left(\gamma_{1 p}+\gamma_{s n}\right) l}{2\left(\gamma_{1 p}+\gamma_{s n}\right)}+\frac{s h\left(\gamma_{1 p}-\gamma_{s n}\right) l}{2\left(\gamma_{1 p}-\gamma_{s n}\right)} \\
& Q_{j n}=\int_{0}^{l} \operatorname{ch} \gamma_{2 j} z^{\prime} \operatorname{ch}\left[\gamma_{3 n}\left(z^{\prime}-l\right)\right] d z^{\prime}=\frac{\gamma_{3 j}}{\gamma_{a j}^{3}-\gamma_{m}^{3}} \operatorname{sh} \gamma_{2 j} l
\end{aligned}
$$

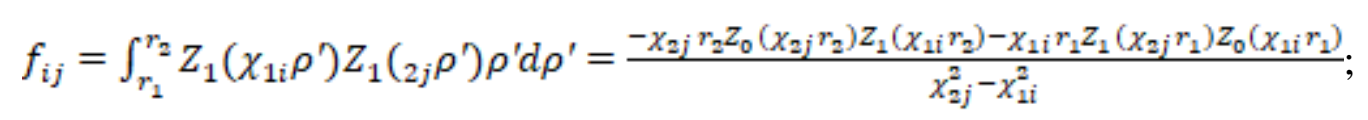

$$
\begin{aligned}
& f_{0 j}=\int_{r_{1}}^{r_{2}} Z_{1}\left(\chi_{10} \rho^{\prime}\right) Z_{1}\left({ }_{2 j} \rho^{\prime}\right) \rho^{s} d \rho^{d} .
\end{aligned}
$$

From equations (9) and (10), it can be seen that the inhomogeneities caused by the change in the cross- section of the line in the planes $z=0$ and $z=l$ are non-interacting. The magnitude of the bias $z=l$ is determined only by the need to reduce the magnitude $C$ and the creation of a consistent inductive reactance.

In practice, the case may occur when $r_{1} \phi r_{2}$. That is, the diameter of the larger of the inner conductors exceeds the diameter of the smaller of the outer conductors. In this case, we obtain such a system of linear algebraic equations for the unknown coefficients of the multiwave scattering matrix:

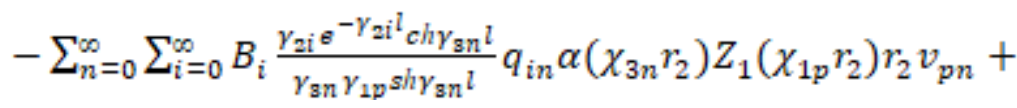

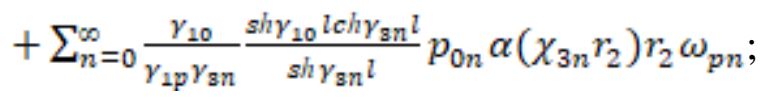




$$
\begin{aligned}
& B_{j}=\sum_{n=0}^{\infty} \sum_{i=0}^{\infty} A_{i} \frac{\gamma_{1 i} e^{-\gamma_{1 i} l} c h \gamma_{3 n} l}{\gamma_{3 n} \gamma_{2 j} \operatorname{sh} \gamma_{3 n} l} p_{i n} \alpha\left(\chi_{3 n} r_{1}\right) Z_{1}\left(\chi_{2 j} r_{1}\right) r_{1} Q_{j n}+ \\
& +\sum_{n=0}^{\infty} \sum_{i=0}^{\infty} B_{i} \frac{\gamma_{2 i} e^{-\gamma_{2 i} l} c h \gamma_{3 n} l}{\gamma_{3 n} \gamma_{2 j} \operatorname{sh} \gamma_{3 n} l} q_{i n} \alpha\left(\chi_{3 n} r_{1}\right) Z_{1}\left(\chi_{2 j} r_{1}\right) r_{1} Q_{j n}- \\
& -\sum_{n=0}^{\infty} 2 \frac{\gamma_{10}}{\gamma_{2 j} \gamma_{3 n}} \frac{\operatorname{sh} \gamma_{10} l c h \gamma_{3 n} l}{s h \gamma_{3 n} l} p_{0 n} \alpha\left(\chi_{3 n} r_{1}\right) Z_{1}\left(\chi_{2 j} r_{1}\right) r_{1} Q_{j n}
\end{aligned}
$$

Calculation of non-reflective step transition in a coaxial waveguide is carried out (Fig. 3). The calculations are performed for the ratio $\frac{r_{2}}{r_{0}}=\frac{r_{a}}{r_{1}}=2.1 \frac{r_{2}}{r_{0}}=\frac{r_{2}}{r_{1}}=4.67$. These calculations allow you to choose the size of the displacement, which provides the minimum value of the reflection coefficient from stepwise inhomogeneity.

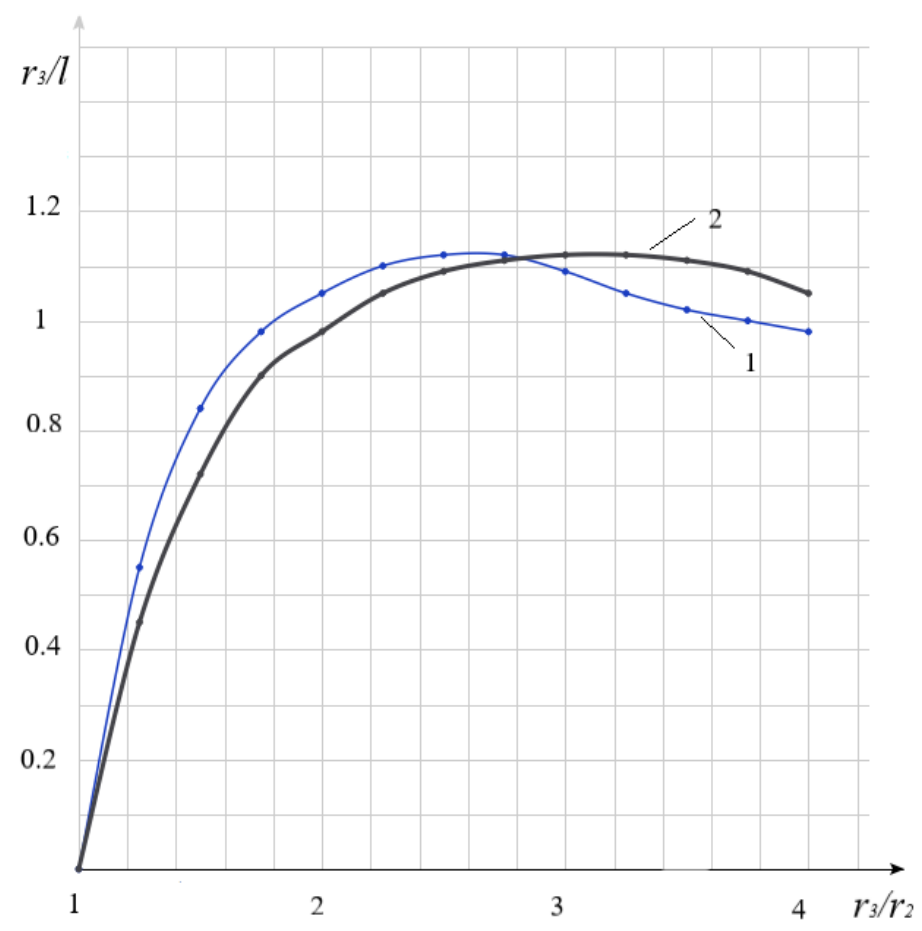

Fig.3. Geometrical dimensions of a step transition providing a minimum of reflection coefficient

\section{Conclusions}

In this paper, inhomogeneities in the form of spasmodic cross-sectional dimensions in a coaxial waveguide are considered.

Using the integral equation method, a stepwise coaxial transition is calculated that minimizes the magnitude of the $\mathrm{T}$-wave reflection coefficient. It is shown that inhomogeneities caused by a change in the cross-section of the line are not interacting.

Calculations results are presented that make it possible to choose the optimal geometric dimensions of such an inhomogeneity. 


\section{References}

1. Dusseaux, R. Telegraphist's equations for rectangular waveguides and analysis in nonorthogonal coordinates / R. Dusseaux, C. Faure. - Progress in Electromagnetics Research. - 2008. - Vol. 88. - P. 53 - 71.

2. Imperiale, S. Mathematical modeling of electromagnetic wave propagation in heterogeneous lossy coaxial cables with variable cross section / S. Imperiale, P Joly / Workshop on Numerical Electromagnetic and Industrial Application (NELIA'2011). Vol. 79. - P. $42-61$.

3. Baida, F.I. Subwavelength metallic coaxial waveguides in the optical range: Role of the plasmonic modes / F.I. Baida, A. Belkhir, D. Van Labeke, O. Lamrous // Physical Review. - 2006. - Vol. 74. - Issue. 20.

4. Sirenko, K.Yu. Splitting of Super-Broadband Pulses by Simple Inhomogeneities of Circular and Coaxial Waveguides / K.Yu. Sirenko // Telecommunications and Radio Engineering. - 2008. - Vol. 67(16). - P.1415-1428.

5. Yu, K.-W. The analysis of a coaxial-to-waveguide transition using FDTD with cylindrical to rectangular cell interpolation scheme / Kyung-Wan Yu, Sung-Choon Kang, Hee-Jin Kang, Jae-Hoon Choi, Jin-Dae Kim // ETRI Journal. - Vol. 21, No. 2. - P. 1 - 8.

6. Hese, J.V. Modeling of discontinuities in general coaxial waveguide structures by the FDTD-method / J.V. Hese, D.D. Zutter // IEEE Transactions on microwave theory and techniques. - 1992. - Vol. 40. - No. 3. - P. $547-556$.

7. Prokhoda, I.G. Dyadic Green's functions and their applications to microwave electromagnetics / I.G. Prokhoda, S.G. Ditruk, V.M. Morozov. - Dnepropetrovsk: DSU. $-1985 .-64 \mathrm{p}$.

8. Gnilenko, A.B. Method of overlapping regions for solving electromagnetic problems / A.B. Gnilenko, V.I. Magro // Proceedings of XXII ${ }^{\text {nd }}$ International Seminar/Workshop on Direct and Inverse Problems of Electromagnetic and Acoustic Wave Theory (DIPED-2017). - Dnipro, Ukraine, 2017. - P. 36 - 41. 